\title{
Stable Cracking Particles Method Based on Stabilized Nodal Integration and Updated Lagrangian Kernel
}

\author{
S. Xu \\ School of Mechanical Engineering, Jiangnan University, China \\ Correspondence should be addressed to S.Xu; ssxu@windowslive.com
}

Received 27 November 2013; Revised 27 January 2014; Accepted 17 February 2014; Published 7 May 2014

Academic Editor: Goangseup Zi

Copyright (C) 2014 S. Xu. This is an open access article distributed under the Creative Commons Attribution License, which permits unrestricted use, distribution, and reproduction in any medium, provided the original work is properly cited.

\begin{abstract}
A stable cracking particles method (CPM) based on updated Lagrangian kernels is proposed. The idea of CPM is to model the crack topology by a set of cracked particles. Hence no representation of the crack surface is needed making the method useful for problems involving complex fracture patterns as they occur in dynamics and under fast loading conditions. For computational efficiency, nodal integration is exploited in the present paper. In order to avoid instabilities, a scheme is presented to stabilized the integration. Moreover, a set of simple cracking rules are proposed in order to prevent numerical fracture. The method is applied to two benchmark problems and shows good accuracy.
\end{abstract}

\section{Introduction}

Meshless methods have been a competitor to finite element method due to their ability to add particles and model large deformations, dynamic fracture, and fragmentation with ease [1-14]. In contrast to finite element methods that require the deletion of elements for complex dynamic fracture problems involving penetration and perforation, meshless methods [14-20] treat such problems quite naturally. However, meshless methods are computationally expensive. Moreover, numerical fracture is reported when fracture and material modelling is not accounted for carefully [21-23]. Recently developed efficient methods such as finite element methods with edge rotations [24-27], phase-field models [28, 29], and partition-of-unity enriched finite element [30-44] and meshless methods have been widely applied to fracture problems with a few number of cracks [23, 45-52]. However, their application to complex dynamic fracture and fragmentation remains a major challenge. A powerful method for complex fracture is the cracking particles method (CPM) $[53,54]$ that is based on enriching nodes with step-enrichment function once a fracture criterion is met. The crack's topology is modeled by discrete plane crack segments and the wellposedness of the initial boundary value problem is restored by means of cohesive zone models. The advantage of the CPM is its robustness and efficiency. Complex fracture is modeled naturally with relatively coarse discretizations as the process zone does not need to be smeared over several particles. The CPM has been applied to numerous challenging problems [55-59]. However, due to the "discontinuous" representation of the crack surface, stresses might be transferred over the opening crack leading to spurious cracking [60-63].

In this paper, we present a stabilized nodal integrated CPM based on updated Lagrangian kernels that alleviates several shortcomings of the original CPM. In particular, the following occurs.

(i) We propose a set of simple cracking rules according to [64] in order to avoid spurious cracking in the CPM. The original CPM shows suffering from spurious cracking adjacent to the crack surface that might artificially increase the dissipated energy.

(ii) We combine for the first time the stabilized nodal integration and the CPM. CPM was only used in combination with computationally expensive Gauss quadrature, stress-point integration and nodal integration. Nodal integration is computationally very efficient but suffers from instabilities. Stress-point integration eliminates these instabilities but cracks are restricted to cross nodes-not stress points. Moreover, there is still the need to update the position of the stress points. Therefore, we employ the stabilized 


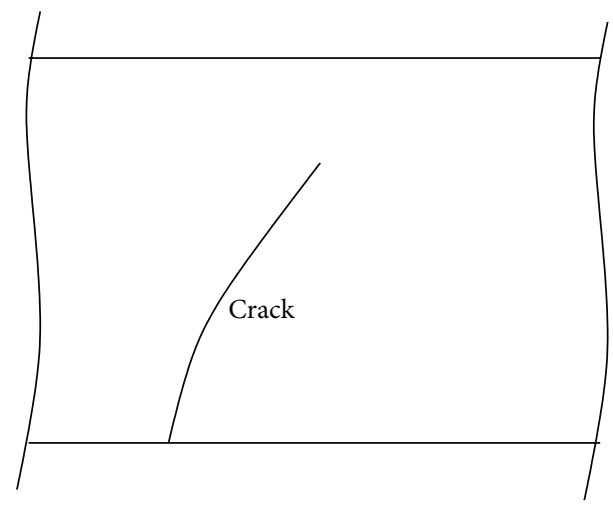

(a) Continuous crack

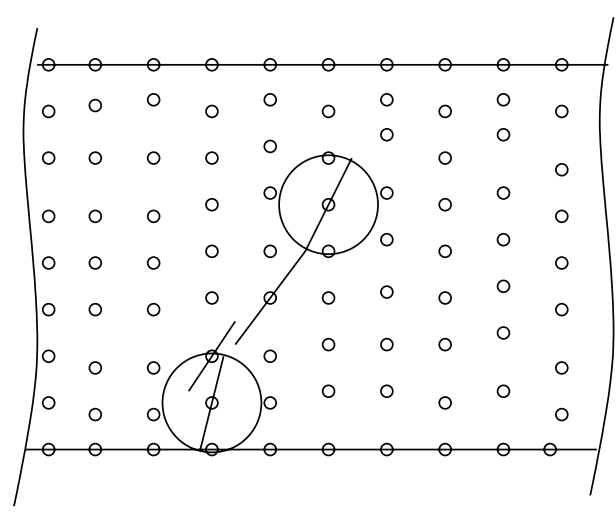

(b) Discretization with crack segments

FIGURE 1: (a) Continuous crack and (b) representation of the crack with discrete cohesive crack segments.

conforming nodal integration [65] also used in the smoothed finite element method [66-70].

(iii) While the original CPM employs a Lagrangian kernel that is only applicable to moderate deformations, an updated Lagrangian kernel formulation is proposed here for the first time. It guarantees the applicability of the method to extremely large deformation.

The paper is organized as follows. First, we present the CPM. Then, the weak form is stated and the discrete equations are derived. Subsequently, the fracture model and the cohesive zone models are discussed before the paper ends with examples and conclusions.

\section{CPM}

The key idea of the CPM is to decompose the displacement field into two parts: the continuous part $\mathbf{u}^{\mathrm{C}}$, sometimes also referred to as the "usual" part, and the discontinuous or "enriched" part $\mathbf{u}^{D}$ :

$$
\mathbf{u}(\mathbf{X}, t)=\mathbf{u}^{C}(\mathbf{X}, t)+\mathbf{u}^{D}(\mathbf{X}, t) .
$$

The discretization of the continuous part of the displacement field is based on moving least squares (MLS) shape functions [71] of linear completeness:

$$
\mathbf{u}^{C}(\mathbf{X}, t)=\sum_{I \in \mathcal{N}} N_{I}(\mathbf{X}) \mathbf{u}_{I}(t)
$$

where $N_{I}(\mathbf{X})$ are the meshless shape functions of node $I$ at position $\mathbf{X}$ and $\mathbf{u}_{I}(t)$ are nodal parameters at time $t$. Note that (1) the nodal parameters are not the true physical displacement values at node $I$ and (2) the shape functions are expressed in terms of material coordinates $\mathbf{X}$. It can be shown that the shape functions are given by

$$
N_{I}(\mathbf{X})=\mathbf{p}^{T}(\mathbf{X}) A^{-1}(\mathbf{X}) \mathbf{D}_{I}(\mathbf{X})
$$

with

$$
\begin{aligned}
& \mathbf{D}_{I}(\mathbf{X})=w\left(\mathbf{X}-\mathbf{X}_{I}, h\right) \mathbf{p}^{T}\left(\mathbf{X}_{I}\right), \\
& \mathbf{A}_{I}(\mathbf{X})=\sum_{I \in \mathcal{N}} w\left(\mathbf{X}-\mathbf{X}_{I}, h\right) \mathbf{p}\left(\mathbf{X}_{I}\right) \mathbf{p}^{T}\left(\mathbf{X}_{I}\right),
\end{aligned}
$$

where $w\left(\mathbf{X}-\mathbf{X}_{I}, h\right)$ denotes the kernel function and $h$ its support size; $\mathbf{p}(\mathbf{X})=(1, X, Y)$ is a linear polynomial basis. We point out again that the kernel function is expressed in terms of material coordinates and therefore is called Lagrangian kernel [22]. It was shown by [22] that a Lagrangian kernel avoids numerical fracture often observed for simulations based on Eulerian kernels. However, the Lagrangian kernel formulation limits the amount of large deformations. Therefore, we update the kernel functions every $n$th time step referring to a new reference configuration and hence call this kernel an updated Lagrangian kernel. A similar approach was mentioned in [54].

The discontinuous part of the displacement field is obtained by simply multiplying the shape function with enrichment functions accounting for the jump in the displacement field (see Figure 2):

$$
\mathbf{u}(\mathbf{X}, t)=\sum_{I \in N_{c}} N_{I}(\mathbf{X}) \mathscr{H}_{\Gamma_{c}}(\mathbf{X}) \mathbf{q}_{I}(t) .
$$

$\mathcal{N}_{c}$ being the set of cracking particles $\mathbf{q}_{I}(t)$ are additional degrees of freedom (DOF) and the enrichment function is the step function

$$
\mathscr{H}(\mathbf{X})=\frac{\mathbf{X} \cdot \mathbf{n}_{\Gamma_{c}}}{\left\|\mathbf{X} \cdot \mathbf{n}_{\Gamma_{c}}\right\|} .
$$

The key strength of the CPM is that it does not require any representation of the crack's topology. In the CPM, the crack topology is described as a set of crack segments as illustrated in Figure 1 allowing the simulation of very complex crack patterns with ease.

It is advantageous to write the approximation of the displacement field in vector matrix notation:

$$
\text { d = ND }
$$

and store the "standard" and enriched shape functions in the matrix N; the DOFs of the "standard" and enriched part of the displacement field are stored in the vector $\mathbf{D}$. Moreover, we can define the matrix $[[\mathbf{N}]]$ and the matrix containing the derivatives of the meshless shape functions $\mathbf{B}$ needed later. 


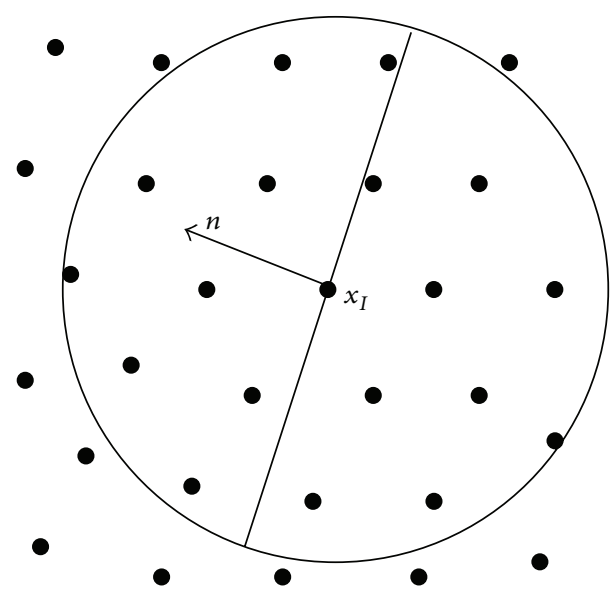

FIGURE 2: Normal of the crack segment.

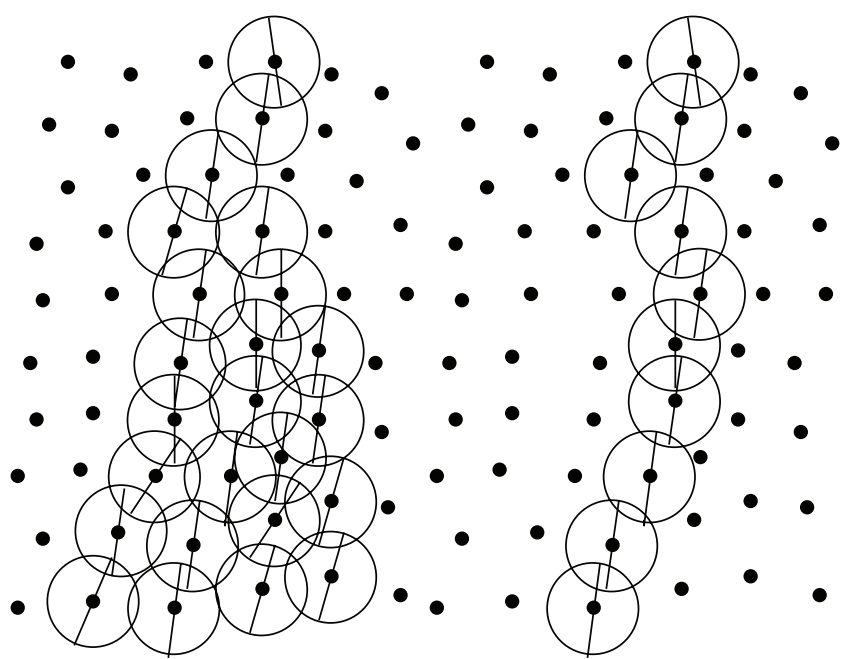

(a)

(b)

FIGURE 3: (a) Spurious cracking during crack propagation and (b) crack propagation without spurious cracking.

Though the CPM can handle complex crack patterns, it might lead to spurious cracking adjacent to the crack that can be avoided by a set of simple rules as shown in [64]. We propose similar rules and firstly distinguish between propagation and initiating cracks by a simple criterion based on a circular support domain. When a cracking particle does not find another cracking particle within the circular domain of size $\alpha h$, then a new crack is initiated. The factor $\alpha$ is chosen to be 1.1 in all simulations. Secondly, we define a zone adjacent to a crack surface where no new cracks can evolve; see Figure 3.

As such a criterion might prevent crack branching, these rules need to be adjusted at the crack tip. Since the crack surface is not continuous in the CPM, we employ the following simple algorithm to detect the crack tip for branching cracks: crack branching occurs when the angle of existing and newly created particles at the crack tip exceeds

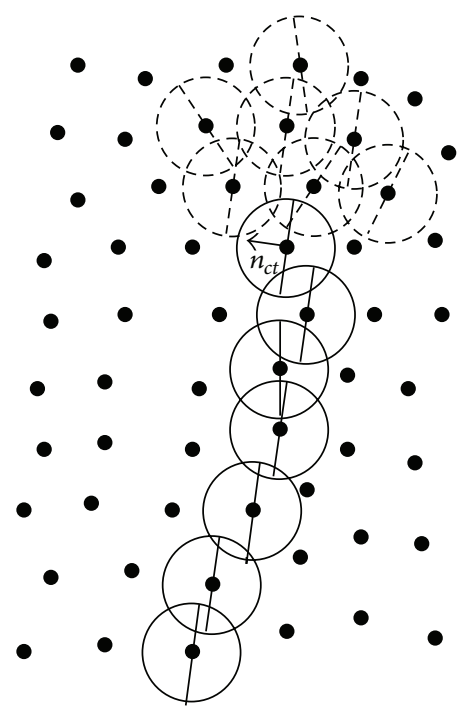

Figure 4: Crack with crack tip node (solid line) at time $n$ and nodes that meet cracking criterion at time $n+1$ (dashed line).

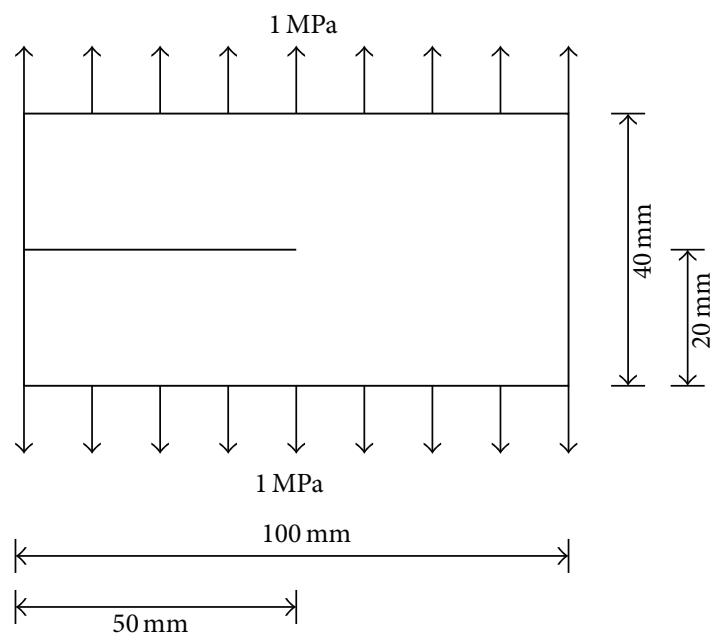

FIgURE 5: Plate with a horizontal initial notch under tensile tractions.

a prescribed tolerance. According to Figure 4, the deviation in this angle is calculated by

$$
\begin{array}{ll}
\gamma_{\max }=\underbrace{\max }_{I \in \widetilde{\mathcal{N}}_{c}}\left(\mathbf{n}_{c t} \cdot \mathbf{n}_{I}\right) & \forall I \in \widetilde{\mathcal{N}}_{c}, \\
\gamma_{\min }=\underbrace{\min }_{I \in \widetilde{\mathcal{N}}_{c}}\left(\mathbf{n}_{c t} \cdot \mathbf{n}_{I}\right) & \forall I \in \widetilde{\mathcal{N}}_{c}
\end{array}
$$

$\widetilde{N}_{c} \subset \mathcal{N}_{c}$ being the set of new cracking particles. Crack branching is assumed when $\gamma_{\max }-\gamma_{\min } \geq$ TOL.

\section{Weak Form and Discretization}

The linear momentum equation is

$$
\nabla \cdot \mathbf{P}+\varrho \mathbf{b}=\varrho \ddot{\mathbf{u}}, \quad \mathbf{X} \in \Omega,
$$




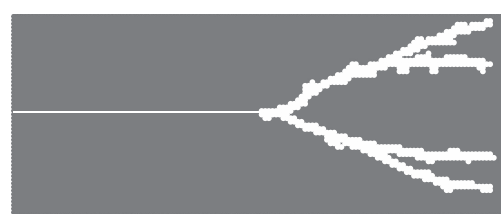

(a) 4141 nodes; with spurious cracking

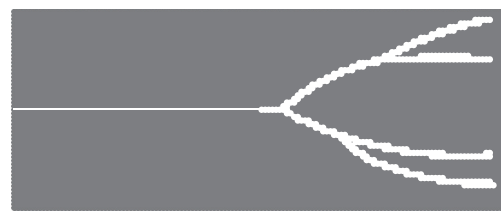

(c) 4141 nodes; without spurious cracking

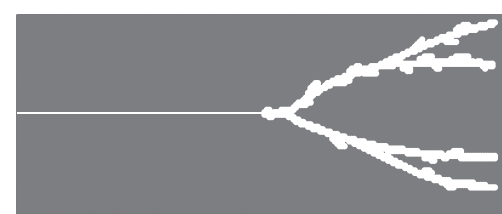

(b) 16281 nodes; with spurious cracking

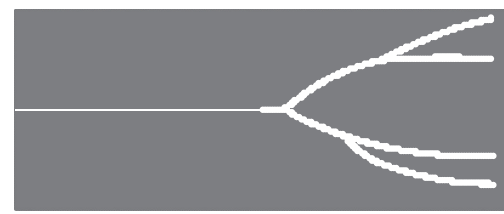

(d) 16281 nodes; without spurious cracking

FIGURE 6: Crack pattern for the crack branching problem.

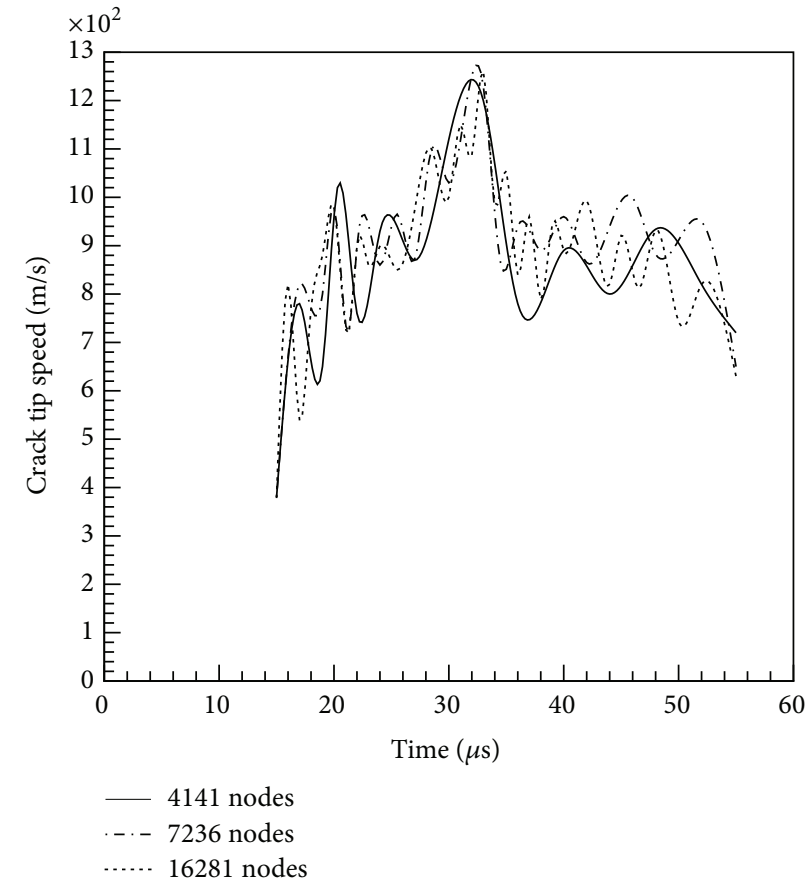

(a) Without spurious cracking

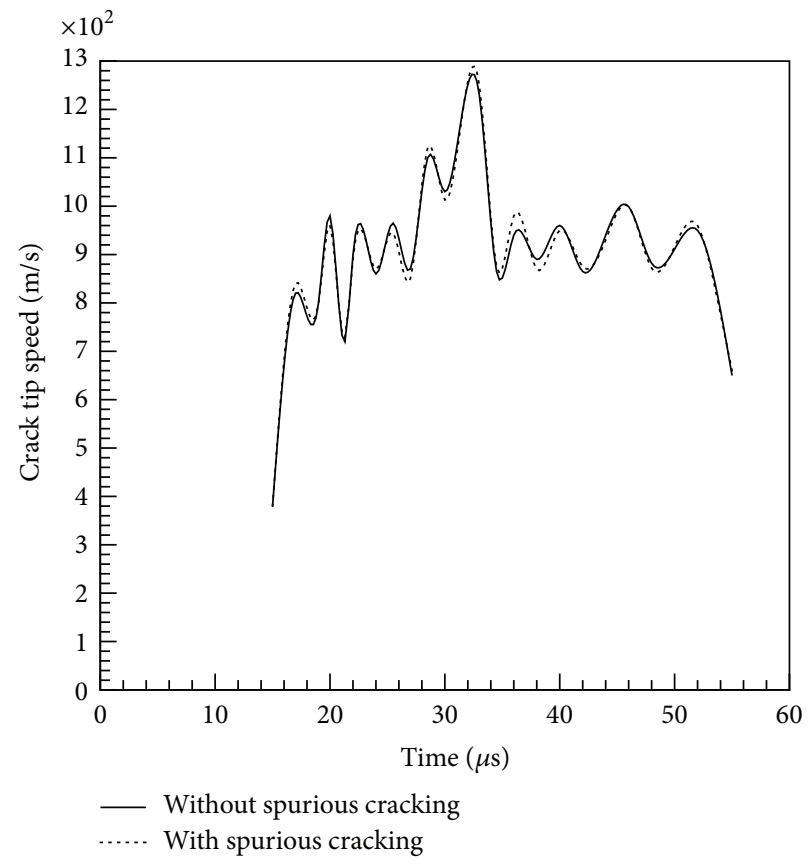

(b) Comparison with and without spurious cracking

FIGURE 7: Crack tip speed for the crack branching problem.

where $\mathbf{P}$ is the nominal stress tensor, $\varrho$ is the density, $\mathbf{b}$ are body forces, and the superimposed dots denote material time derivatives. The displacement and traction boundary conditions are

$$
\begin{aligned}
\mathbf{u} & =\overline{\mathbf{u}}, \quad \mathbf{X} \in \Gamma_{u} \\
\mathbf{n}_{t} \cdot \mathbf{P} & =\overline{\mathbf{t}}, \quad \mathbf{X} \in \Gamma_{t} \\
\mathbf{n}_{c} \cdot \mathbf{P} & =\mathbf{t}_{c}([[\mathbf{u}]]), \quad \mathbf{X} \in \Gamma_{c},
\end{aligned}
$$

where the index $c$ refers to the crack, the index $t$ refers to traction boundaries, and the index $u$ refers to displacement boundaries.
We solve the equation of motion in weak form that can be stated in variational form. Find the displacement field $\mathbf{u} \epsilon$ $\mathscr{U} \forall \delta \mathbf{u} \in \mathscr{V}$ such that the first variation in the energy is zero:

$$
\delta W=\delta W_{\text {int }}-\delta W_{\text {ext }}+\delta W_{\text {inertia }}-\delta W_{\text {coh }}=0
$$

$$
\forall \delta \mathbf{u} \in \mathscr{U}_{0}
$$

with

$$
\begin{aligned}
& \delta W_{\mathrm{int}}=\int_{\Omega} \nabla \delta \mathbf{u}: \mathbf{P} d \Omega, \\
& \delta W_{\mathrm{ext}}=\int_{\Gamma_{t}} \delta \mathbf{u} \cdot \overline{\mathbf{t}} d \Gamma+\int_{\Omega} \varrho \delta \mathbf{u} \cdot \mathbf{b} d \Omega,
\end{aligned}
$$




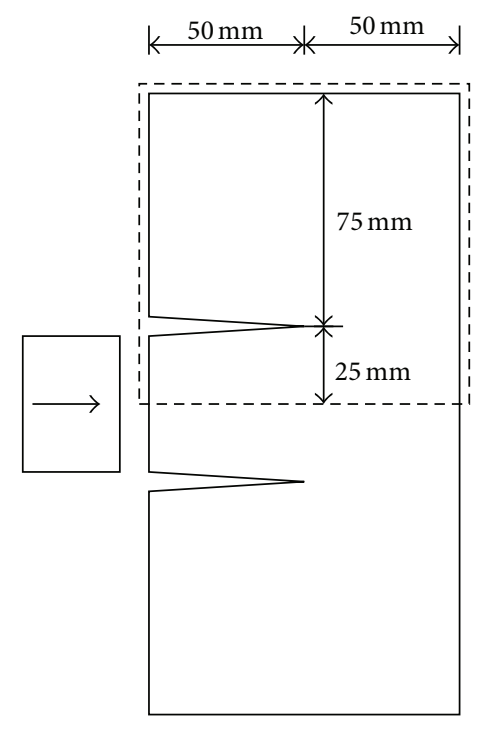

FIgURE 8: The Kalthoff problem.

$$
\begin{aligned}
\delta W_{\text {inertia }} & =\int_{\Omega} \varrho \delta \mathbf{u} \cdot \ddot{\mathbf{u}} d \Omega, \\
\delta W_{\text {coh }} & =\int_{\Gamma_{c}} \delta[[\mathbf{u}]] \cdot \mathbf{t}_{c} d \Gamma .
\end{aligned}
$$

The approximation spaces $\mathcal{U}$ and $\mathcal{U}_{0}$ are given by

$$
\begin{gathered}
\mathcal{U}=\left\{\mathbf{u}(\mathbf{X}, t) \mid \mathbf{u} \in H^{1}, \mathbf{u}=\overline{\mathbf{u}} \text { on } \Gamma_{u},\right. \\
\left.\mathbf{u} \text { discontinuous on } \Gamma_{c}\right\}, \\
\mathcal{U}_{0}=\left\{\delta \mathbf{u} \mid \delta \mathbf{u} \in H^{1}, \delta \mathbf{u}=0 \text { on } \Gamma_{u},\right. \\
\left.\delta \mathbf{u} \text { discontinuous on } \Gamma_{c}\right\} .
\end{gathered}
$$
by

The essential and natural boundary conditions are given

$$
\begin{aligned}
\mathbf{u} & =\overline{\mathbf{u}}, \quad \mathbf{X} \in \Gamma_{u} \\
\mathbf{n}_{t} \cdot \mathbf{P} & =\overline{\mathbf{t}}, \quad \mathbf{X} \in \Gamma_{t} \\
\mathbf{n}_{c} \cdot \mathbf{P} & =\mathbf{t}_{c}([[\mathbf{u}]]), \quad \mathbf{X} \in \Gamma_{c},
\end{aligned}
$$

the index $c$ referring to crack boundaries, the index $t$ to natural boundaries, and the index $u$ to essential boundaries.

By substituting the approximation of the displacement field $\mathbf{u}$ and the virtual displacement field $\delta \mathbf{u}$ into the weak form (11), the discrete system of equations is obtained:

$$
\begin{aligned}
\sum_{J=1}^{n} \int_{\Omega_{J}} \nabla \delta \mathbf{u}_{J} & : \mathbf{P} d \Omega-\sum_{J=1}^{n} \int_{\Gamma_{t, J}} \delta \mathbf{u} \cdot \overline{\mathbf{t}} d \Gamma \\
& -\sum_{J=1}^{n} \int_{\Omega_{J}} \varrho \delta \mathbf{u} \cdot \mathbf{b} d \Omega+\int_{\Gamma_{c, J}} \delta[[\mathbf{u}]] \cdot \mathbf{t}_{c} d \Gamma \\
& +\sum_{J=1}^{n} \int_{\Omega_{J}} \varrho \delta \mathbf{u} \cdot \ddot{\mathbf{u}} d \Omega=0
\end{aligned}
$$

or in vector-matrix form:

$$
\mathbf{M}_{I J} \mathbf{D}_{J}=\mathbf{F}_{I}^{\mathrm{ext}}-\mathbf{F}_{I}^{\mathrm{int}}
$$

with

$$
\begin{gathered}
\mathbf{F}_{I}^{\text {ext }}=\int_{\Gamma_{t}}\left(\mathbf{N}_{I}\right)^{T} \mathbf{t} d \Gamma+\int_{\Omega}\left(\mathbf{N}_{I}\right)^{T} \mathbf{b} d \Omega+\int_{\Gamma_{c}}\left[\left[\left(\mathbf{N}_{I}\right)^{T}\right]\right] \mathbf{t}_{c} d \Gamma, \\
\mathbf{F}_{I}^{\text {int }}=\int_{\Omega}\left(\mathbf{B}_{I}\right)^{T} \mathbf{P} d \Omega, \\
\mathbf{M}_{I J}=\int_{\Omega} \varrho \mathbf{N}_{I} \mathbf{N}_{J}^{T} d \Omega .
\end{gathered}
$$

For computational efficiency, nodal integration is employed. Nodal integration does not drastically reduce the number of quadrature points; it was shown, for example, by [72] that the critical time step is increased by orders of magnitude; care has to be taken in the existence of cracks [73]. However, nodal integration leads to instabilities due to rank deficiency. Therefore, we employ the stabilized conforming nodal integration technique as mentioned previously. Stabilized conforming nodal integration has also shown great performance for fracture problems in the context of the finite element method; see [74-80]. The explicit central difference time integration scheme is used (for the time integration).

\section{Fracture Criterion and Cohesive Zone Model}

Fracture is governed by the maximum principal stress. The crack is introduced perpendicular to the direction of the maximum principal stress. Complex crack patterns are obtained automatically as the method does not require any representation of the crack's topology. There is no need to distinguish between crack nucleation and crack propagation and complex crack patterns including crack branching are natural outcome of the simulation.

The cohesive zone model related the jump in the displacement field $[[\mathbf{u}]]$ to the cohesive traction at the crack surface. The discontinuous displacement field can be split into a part acting perpendicular and another tangential to the crack surface:

$$
\begin{aligned}
& \delta_{n}=[[\mathbf{u}]] \cdot \mathbf{n} \\
& \delta_{t}=\left|[[\mathbf{u}]]-\delta_{n} \mathbf{n}\right|
\end{aligned}
$$

Defining an effective crack opening displacement as suggested by [81]

$$
\delta=\sqrt{\beta^{2} \delta_{t}^{2}-\delta_{n}^{2}}
$$

an effective cohesive zone model can be derived by

$$
t=\frac{t_{\max }}{\delta_{\max }} \delta \quad \text { if } \delta \leq \delta_{\max } \text { or } \dot{\delta}<0 .
$$




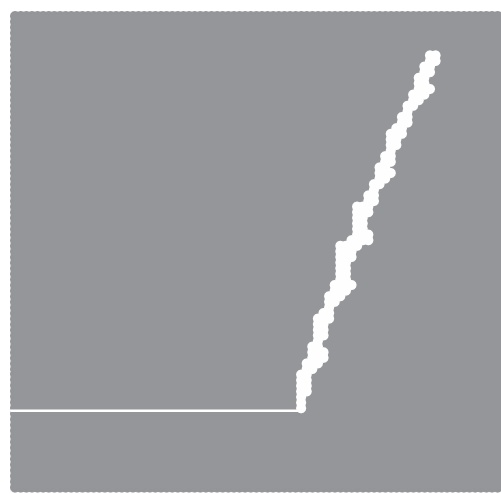

(a) 10201 nodes; with spurious cracking

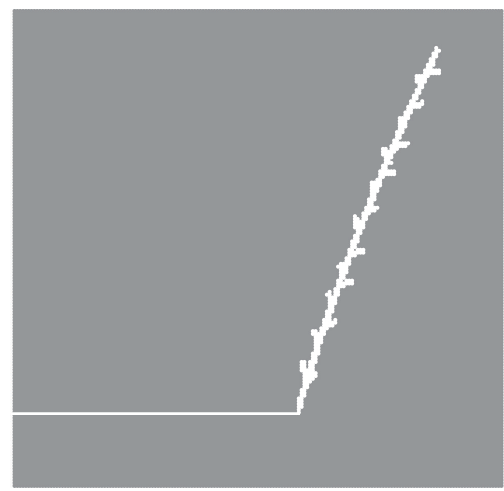

(c) 40804 nodes; with spurious cracking

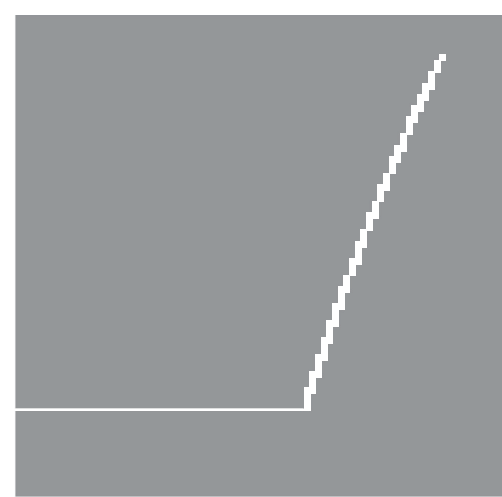

(b) 10201 nodes; without spurious cracking

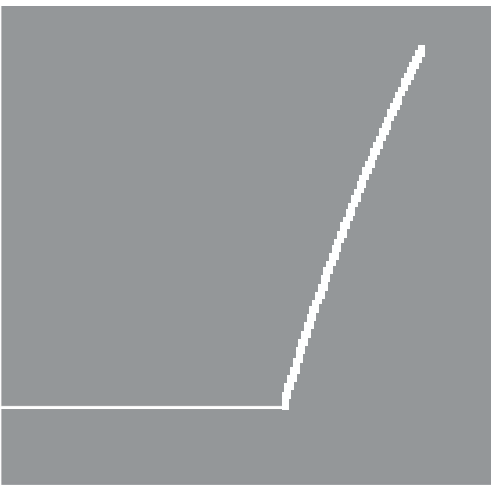

(d) 40804 nodes; without spurious cracking

FIGURE 9: Final crack path of the Kalthoff problem.

The traction vector is then obtained by

$$
\mathbf{t}=\frac{t}{\delta}\left(\beta^{2} \delta_{t}+\delta_{n} \mathbf{n}\right)
$$

where $\beta$ determines the amount of tangential and normal tractions.

\section{Results}

5.1. Crack Branching. Let us consider a specimen with initial crack as illustrated in Figure 5. It is subjected to uniaxial tensile loading of $\sigma=1 \mathrm{MPa}$ at the top and bottom. This classical benchmark problem of dynamic fracture has been studied by several people to test the accuracy and robustness of their computational method [39, 53, 82, 83]. Also, experimental data can be found for such type of problems; see, for example, [84-86]. The material properties for this example are modulus of elasticity $E=32,000 \mathrm{MPa}$ and Poisson's ratio $v=0.20$. The maximum crack speed is restricted by the Rayleigh wave speed $c_{R}=2119.0 \mathrm{~m} / \mathrm{s}$. Discretizations ranging from only 4000 nodes up to more than 16000 nodes are investigated. Also the influence of the cracking rules will be demonstrated in this section.

The fracture patterns at various time steps are depicted in Figure 6. Spurious cracks adjacent to the "main" crack are observed when no cracking rules are applied. The velocity of the propagating crack is illustrated in Figure 7. We note the following.

(i) The cracking rules do not influence the crack speed indicating that much less energy is dissipated in the spurious cracks.

(ii) The crack propagates faster just before it branches.

(iii) The maximum crack speed is far below the Raleigh wave speed. This agrees well with experimental observations of the microbranch instability problem as reported by [84-86]. The ability to capture this physical behaviour naturally is one advantage of the CPM over other methods with continuous crack surface $[23,39,51,87]$.

5.2. The Kalthoff Experiment. The second classical benchmark example studied here is the Kalthoff problem [88]. Therefore consider the double-notched specimen under impact loading $(v=20 \mathrm{~m} / \mathrm{s})$ as illustrated in Figure 8 . The impact leads to mode I dominated fracture with a crack propagating almost orthogonal to the impact loading.

We exploit the symmetry of the model and carry out simulations of discretizations ranging from 10,000 to 40,000 particles. According to [89], the modulus of elasticity in these experiments is $E=190 \mathrm{GPa}$, the initial density is $\varrho_{0}=$ $8000 \mathrm{~kg} / \mathrm{m}^{3}$, and Poisson's ratio is $v=0.3$. Figure 9 shows the final fracture pattern that matches the experimental 


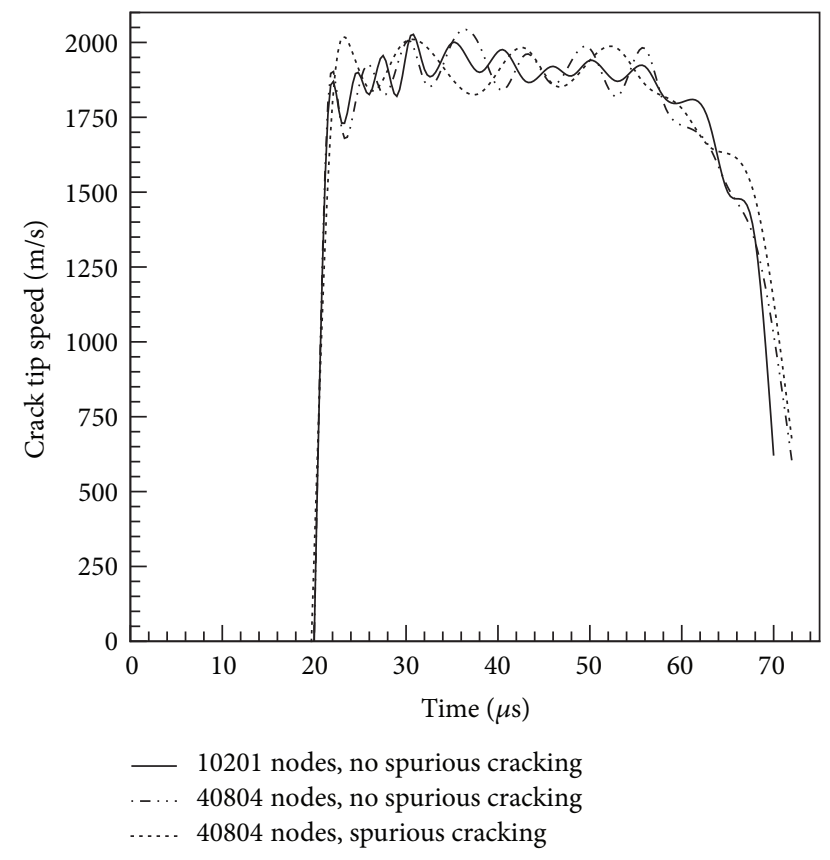

Figure 10: Crack tip speed and cohesive fracture energy of the Kalthoff problem.

data. Figure 10 reveals that the crack speed here is also not influenced by the cracking rules. However, the crack looks more erratic without using the cracking rules.

\section{Conclusions}

In conclusion, a cracking particles method based on nodal integration and updated Lagrangian kernels was proposed where a set of simple cracking rules was suggested in order to avoid spurious cracking. The stabilized nodal integration guarantees the computational efficiency of the method while maintaining the stability. Moreover, the updated Lagrangian kernel ensures that the method remains applicable also for extremely large deformations.

The method was applied to two benchmark examples where the performance of the method was demonstrated.

\section{Conflict of Interests}

The author declares that there is no conflict of interests regarding the publication of this paper.

\section{References}

[1] S. Li and W. K. Liu, "Meshfree and particle methods and their applications," Applied Mechanics Reviews, vol. 55, no. 1, pp.1-34, 2002.

[2] S. Li and W. K. Liu, Meshfree Particle Methods, Springer, 2004.

[3] S. Li and D. C. Simkins Jr., "Conserving Galerkin weak formulations for computational fracture mechanics," Communications in Numerical Methods in Engineering, vol. 18, no. 12, pp. 835-850, 2002.
[4] S. Li and B. C. Simonson, "Meshfree simulation of ductile crack propagation," International Journal of Computational Methods in Engineering Science and Mechanics, vol. 6, pp. 1-19, 2003.

[5] S. Li and B. C. Simonson, "Meshfree simulation of ductile crack propagation," International Journal of Computational Methods in Engineering Science and Mechanics, vol. 60, no. 8, pp. 1425$1450,2004$.

[6] S. Li, W. Hao, and W. K. Liu, "Mesh-free simulations of shear banding in large deformation," International Journal of Solids and Structures, vol. 37, no. 48, pp. 7185-7206, 2000.

[7] S. Li, W. Hao, and W. K. Liu, "Numerical simulations of large deformation of thin shell structures using meshfree methods," Computational Mechanics, vol. 25, no. 2, pp. 102-116, 2000.

[8] S. Li, W. K. Liu, A. J. Rosakis, T. Belytschko, and W. Hao, "Meshfree Galerkin simulations of dynamic shear band propagation and failure mode transition," International Journal of Solids and Structures, vol. 39, no. 5, pp. 1213-1240, 2002.

[9] A. G. Petschek and L. D. Libersky, "Cylindrical smoothed particle hydrodynamics," Journal of Computational Physics, vol. 109, no. 1, pp. 76-83, 1993.

[10] T. Rabczuk and J. Eibl, "Modelling dynamic failure of concrete with meshfree methods," International Journal of Impact Engineering, vol. 32, no. 11, pp. 1878-1897, 2006.

[11] T. Rabczuk and J. Eibl, "Simulation of high velocity concrete fragmentation using SPH/MLSPH," International Journal for Numerical Methods in Engineering, vol. 56, no. 10, pp. 1421-1444, 2003.

[12] T. Rabczuk, J. Eibl, and L. Stempniewski, "Numerical analysis of high speed concrete fragmentation using a meshfree Lagrangian method," Engineering Fracture Mechanics, vol. 71, no. 4-6, pp. 547-556, 2004.

[13] T. Rabczuk, S. P. Xiao, and M. Sauer, "Coupling of mesh-free methods with finite elements: Basic concepts and test results," Communications in Numerical Methods in Engineering, vol. 22, no. 10, pp. 1031-1065, 2006.

[14] P. W. Randles and L. D. Libersky, "Recent improvements in SPH modeling of hypervelocity impact," International Journal of Impact Engineering, vol. 20, no. 6-10, pp. 525-532, 1997.

[15] T. Belytschko, Y. Y. Lu, L. Gu, and M. Tabbara, "Element-free galerkin methods for static and dynamic fracture," International Journal of Solids and Structures, vol. 32, no. 17-18, pp. 2547-2570, 1995.

[16] T. Belytschko, Y. Krongauz, D. Organ, M. Fleming, and P. Krysl, "Meshless methods: an overview and recent developments," Computer Methods in Applied Mechanics and Engineering, vol. 139, no. 1-4, pp. 3-47, 1996.

[17] W. K. Liu, S. J. Jun, and Y. F. Zhang, "Reproducing kernel particle methods," International Journal for Numerical Methods in Fluids, vol. 20, no. 8-9, pp. 1081-1106, 1995.

[18] V. P. Nguyen, T. Rabczuk, S. Bordas, and M. Duflot, "Meshless methods: a review and computer implementation aspects," Mathematics and Computers in Simulation, vol. 79, no. 3, pp. 763-813, 2008.

[19] T. Rabczuk and T. Belytschko, "Adaptivity for structured meshfree particle methods in 2D and 3D," International Journal for Numerical Methods in Engineering, vol. 63, no. 11, pp. 1559-1582, 2005.

[20] P. W. Randles and L. D. Libersky, "Normalized SPH with stress points," International Journal for Numerical Methods in Engineering, vol. 48, no. 10, pp. 1445-1462, 2000. 
[21] T. Belytschko, Y. Guo, W. K. Liu, and S. P. Xiao, "A unified stability analysis of meshless particle methods," International Journal for Numerical Methods in Engineering, vol. 48, no. 9, pp. 1359-1400, 2000.

[22] T. Rabczuk, T. Belytschko, and S. P. Xiao, "Stable particle methods based on Lagrangian kernels," Computer Methods in Applied Mechanics and Engineering, vol. 193, no. 12-14, pp. 10351063, 2004.

[23] T. Rabczuk, S. Bordas, and G. Zi, "A three dimensional meshfree method for static and dynamic multiple crack nucleation/propagation with crack path continuity," Computational Mechanics, vol. 40, no. 3, pp. 473-495, 2007.

[24] P. Areias and T. Rabczuk, "Finite strain fracture of plates and shells with configurational forces and edge rotations," International Journal for Numerical Methods in Engineering, vol. 94, no. 12, pp. 1099-1122, 2013.

[25] P. Areias, D. Dias da Costa, J. M. Sargado, and T. Rabczuk, "Element-wise algorithm for modeling ductile fracture with the rousselier yield function," Computational Mechanics, vol. 52, no. 6, pp. 1429-1443, 2013.

[26] P. Areias, T. Rabczuk, and P. P. Camanho, "Initially rigid cohesive laws and fracture based on edge rotations," Computational Mechanics, vol. 52, no. 4, pp. 931-947, 2013.

[27] P. Areias, T. Rabczuk, and D. Dias da Costa, "Element-wise fracture algorithm based on rotation of edges," Engineering Fracture Mechanics, vol. 110, pp. 113-137, 2013.

[28] C. Miehe, M. Hofacker, and F. Welschinger, "A phase field model for rate-independent crack propagation: Robust algorithmic implementation based on operator splits," Computer Methods in Applied Mechanics and Engineering, vol. 199, no. 45-48, pp. 2765-2778, 2010.

[29] C. Miehe, F. Welschinger, and M. Hofacker, "Thermodynamically consistent phase-field models of fracture: variational principles and multi-field FE implementations," International Journal for Numerical Methods in Engineering, vol. 83, no. 10, pp. 1273-1311, 2010.

[30] F. Armero and K. Garikipati, "Recent advances in the analysis and numericalsimulation of strain localization in inelastic solids," in Proceedings of the 4th Computational Plasticity: Fundamentals and Applications Conference (COMPLAS '00), pp. 635-640, 2000.

[31] T. Belytschko and T. Black, "Elastic crack growth in finite elements with minimal remeshing," International Journal for Numerical Methods in Engineering, vol. 45, no. 5, pp. 601-620, 1999.

[32] T. Belytschko, H. Chen, J. Xu, and G. Zi, "Dynamic crack propagation based on loss of hyperbolicity and a new discontinuous enrichment," International Journal for Numerical Methods in Engineering, vol. 58, no. 12, pp. 1873-1905, 2003.

[33] T. Chau-Dinh, G. Zi, P.-S. Lee, T. Rabczuk, and J.-H. Song, "Phantom-node method for shell models with arbitrary cracks," Computers \& Structures, vol. 92-93, pp. 242-246, 2012.

[34] G. A. Dilts, "Moving-least-squares-particle hydrodynamics. I. Consistency and stability," International Journal for Numerical Methods in Engineering, vol. 44, no. 8, pp. 1115-1155, 1999.

[35] G. A. Dilts, "Moving least-squares particle hydrodynamics. II. Conservation and boundaries," International Journal for Numerical Methods in Engineering, vol. 48, no. 10, pp.1503-1524, 2000.

[36] C. Linder and F. Armero, "Finite elements with embedded strong discontinuities for the modeling of failure in solids,"
International Journal for Numerical Methods in Engineering, vol. 72, no. 12, pp. 1391-1433, 2007.

[37] N. Moës, J. Dolbow, and T. Belytschko, "A finite element method for crack growth without remeshing," International Journal for Numerical Methods in Engineering, vol. 46, no. 1, pp. 131-150, 1999.

[38] S. S. Nanthakumar, T. Lahmer, and T. Rabczuk, "Detection of flaws in piezoelectric structures using extended FEM," International Journal for Numerical Methods in Engineering, vol. 96, no. 6, pp. 373-389, 2013.

[39] J.-H. Song, P. M. A. Areias, and T. Belytschko, "A method for dynamic crack and shear band propagation with phantom nodes," International Journal for Numerical Methods in Engineering, vol. 67, no. 6, pp. 868-893, 2006.

[40] T. Strouboulis, K. Copps, and I. Babuška, "The generalized finite element method: An example of its implementation and illustration of its performance," International Journal for Numerical Methods in Engineering, vol. 47, no. 8, pp. 1401-1417, 2000.

[41] G. Ventura, E. Budyn, and T. Belytschko, "Vector level sets for description of propagating cracks in finite elements," International Journal for Numerical Methods in Engineering, vol. 58, no. 10, pp. 1571-1592, 2003.

[42] G. Zi and T. Belytschko, "New crack-tip elements for XFEM and applications to cohesive cracks," International Journal for Numerical Methods in Engineering, vol. 57, no. 15, pp. 2221-2240, 2003.

[43] G. Zi, J.-H. Song, E. Budyn, S.-H. Lee, and T. Belytschko, "A method for growing multiple cracks without remeshing and its application to fatigue crack growth," Modelling and Simulation in Materials Science and Engineering, vol. 12, no. 5, pp. 901-915, 2004.

[44] G. Zi, H. Chen, J. Xu, and T. Belytschko, "The extended finite element method for dynamic fractures," Shock and Vibration, vol. 12, no. 1, pp. 9-23, 2005.

[45] F. Amiri, C. Anitescu, M. Arroyo, S. P. A. Bordas, and T. Rabczuk, "XLME interpolants, a seamless bridge between XFEM and enriched meshless methods," Computational Mechanics, vol. 53, no. 1, pp. 45-57, 2014.

[46] S. Bordas, T. Rabczuk, and G. Zi, “Three-dimensional crack initiation, propagation, branching and junction in non-linear materials by an extended meshfree method without asymptotic enrichment," Engineering Fracture Mechanics, vol. 75, no. 5, pp. 943-960, 2008.

[47] T. Rabczuk and P. Areias, "A meshfree thin shell for arbitrary evolving cracks based on an extrinsic basis," Computer Modeling in Engineering and Sciences, vol. 16, no. 2, pp. 115-130, 2006.

[48] T. Rabczuk, G. Zi, S. Bordas, and H. Nguyen-Xuan, "A geometrically non-linear three-dimensional cohesive crack method for reinforced concrete structures," Engineering Fracture Mechanics, vol. 75, no. 16, pp. 4740-4758, 2008.

[49] T. Rabczuk, S. Bordas, and G. Zi, "On three-dimensional modelling of crack growth using partition of unity methods," Computers \& Structures, vol. 88, no. 23-24, pp. 1391-1411, 2010.

[50] T. Rabczuk, R. Gracie, J.-H. Song, and T. Belytschko, "Immersed particle method for fluid-structure interaction," International Journal for Numerical Methods in Engineering, vol. 81, no. 1, pp. 48-71, 2010.

[51] G. Ventura, J. X. Xu, and T. Belytschko, "A vector level set method and new discontinuity approximations for crack growth by EFG," International Journal for Numerical Methods in Engineering, vol. 54, no. 6, pp. 923-944, 2002. 
[52] G. Zi, T. Rabczuk, and W. Wall, "Extended meshfree methods without branch enrichment for cohesive cracks," Computational Mechanics, vol. 40, no. 2, pp. 367-382, 2007.

[53] T. Rabczuk and T. Belytschko, "Cracking particles: a simplified meshfree method for arbitrary evolving cracks," International Journal for Numerical Methods in Engineering, vol. 61, no. 13, pp. 2316-2343, 2004.

[54] T. Rabczuk and T. Belytschko, "A three-dimensional large deformation meshfree method for arbitrary evolving cracks," Computer Methods in Applied Mechanics and Engineering, vol. 196, no. 29-30, pp. 2777-2799, 2007.

[55] T. Rabczuk and P. M. A. Areias, "A new approach for modelling slip lines in geological materials with cohesive models," International Journal for Numerical and Analytical Methods in Geomechanics, vol. 30, no. 11, pp. 1159-1172, 2006.

[56] T. Rabczuk and T. Belytschko, "Application of particle methods to static fracture of reinforced concrete structures," International Journal of Fracture, vol. 137, no. 1-4, pp. 19-49, 2006.

[57] T. Rabczuk and E. Samaniego, "Discontinuous modelling of shear bands using adaptive meshfree methods," Computer Methods in Applied Mechanics and Engineering, vol. 197, no. 6-8, pp. 641-658, 2008.

[58] T. Rabczuk, P. M. A. Areias, and T. Belytschko, "A meshfree thin shell method for non-linear dynamic fracture," International Journal for Numerical Methods in Engineering, vol. 72, no. 5, pp. 524-548, 2007.

[59] T. Rabczuk, J.-H. Song, and T. Belytschko, "Simulations of instability in dynamic fracture by the cracking particles method," Engineering Fracture Mechanics, vol. 76, no. 6, pp. 730-741, 2009.

[60] L. Chen and Y. Zhang, "Dynamic fracture analysis using discrete cohesive crack method," International Journal for Numerical Methods in Biomedical Engineering, vol. 26, no. 11, pp. 1493$1502,2010$.

[61] Y. Dong, S. Wu, S. S. Xu, Y. Zhang, and S. Fang, "Analysis of concrete fracture using a novel cohesive crack method," Applied Mathematical Modelling, vol. 34, no. 12, pp. 4219-4231, 2010.

[62] N. Sageresan and R. Drathi, "Crack propagation in concrete using meshless method," Computer Modeling in Engineering and Sciences, vol. 32, no. 2, pp. 103-112, 2008.

[63] H. X. Wang and S. X. Wang, "Analysis of dynamic fracture with cohesive crack segment method," Computer Modeling in Engineering \& Sciences, vol. 35, no. 3, pp. 253-274, 2008.

[64] T. Rabczuk, G. Zi, S. Bordas, and H. Nguyen-Xuan, "A simple and robust three-dimensional cracking-particle method without enrichment," Computer Methods in Applied Mechanics and Engineering, vol. 199, no. 37-40, pp. 2437-2455, 2010.

[65] J. S. Chen, C. T. Wu, S. Yoon, and Y. You, "A stabilized conforming nodal integration for galerkin meshfree-methods," International Journal for Numerical Methods in Engineering, vol. 50, pp. 435-466, 2001.

[66] G. R. Liu, T. T. Nguyen, K. Y. Dai, and K. Y. Lam, “Theoretical aspects of the smoothed finite element method (SFEM)," International Journal for Numerical Methods in Engineering, vol. 71, no. 8, pp. 902-930, 2007.

[67] T. Nguyen-Thoi, H. C. Vu-Do, T. Rabczuk, and H. NguyenXuan, "A node-based smoothed finite element method (NSFEM) for upper bound solution to visco-elastoplastic analyses of solids using triangular and tetrahedral meshes," Computer Methods in Applied Mechanics and Engineering, vol. 199, no. 4548, pp. 3005-3027, 2010.
[68] T. Nguyen-Thoi, P. Phung-Van, T. Rabczuk, H. Nguyen-Xuan, and C. Le-Van, "Free and forced vibration analysis using the n-sided polygonal cellbased smoothed finite element method (ncs-fem)," International Journal of Computational Methods, vol. 10, no. 1, Article ID 13400082, 2013.

[69] H. Nguyen-Xuan, T. Rabczuk, T. Nguyen-Thoi, T. N. Tran, and N. Nguyen-Thanh, "Computation of limit and shakedown loads using a node-based smoothed finite element method," International Journal for Numerical Methods in Engineering, vol. 90, no. 3, pp. 287-310, 2012.

[70] C. H. Thai, H. Nguyen-Xuan, N. Nguyen-Thanh, T.-H. Le, T. Nguyen-Thoi, and T. Rabczuk, "Static, free vibration, and buckling analysis of laminated composite Reissner-Mindlin plates using NURBS-based isogeometric approach," International Journal for Numerical Methods in Engineering, vol. 91, no. 6, pp. 571-603, 2012.

[71] T. Belytschko, Y. Y. Lu, and L. Gu, "Element-free Galerkin methods," International Journal for Numerical Methods in Engineering, vol. 37, no. 2, pp. 229-256, 1994.

[72] M. A. Puso, J. S. Chen, E. Zywicz, and W. Elmer, "Meshfree and finite element nodal integration methods," International Journal for Numerical Methods in Engineering, vol. 74, no. 3, pp. 416446, 2008.

[73] H. Talebi, C. Samaniego, E. Samaniego, and T. Rabczuk, "On the numerical stability and mass-lumping schemes for explicit enriched meshfree methods," International Journal for Numerical Methods in Engineering, vol. 89, no. 8, pp. 1009-1027, 2012.

[74] P. Baiz, S. Natarajan, S. Bordas, P. Kerfriden, and T. Rabczuk, "Linear buckling analysis of cracked plates by sfem and xfem (smxfem)," Journal of Mechanics of Materials and Structures, vol. 6, no. 9-10, pp. 1213-1238, 2011.

[75] S. P. A. Bordas, T. Rabczuk, N.-X. Hung et al., "Strain smoothing in FEM and XFEM," Computers \& Structures, vol. 88, no. 23-24, pp. 1419-1443, 2010.

[76] S. P. A. Bordas, S. Natarajan, P. Kerfriden et al., "On the performance of strain smoothing for quadratic and enriched finite element approximations (XFEM/GFEM/PUFEM)," International Journal for Numerical Methods in Engineering, vol. 86, no. 4-5, pp. 637-666, 2011.

[77] L. Chen, T. Rabczuk, S. P. A. Bordas, G. R. Liu, K. Y. Zeng, and P. Kerfriden, "Extended finite element method with edge-based strain smoothing (ESm-XFEM) for linear elastic crack growth," Computer Methods in Applied Mechanics and Engineering, vol. 212, no. 4, pp. 250-265, 2012.

[78] H. Nguyen-Xuan, G. R. Liu, S. Bordas, S. Natarajan, and T. Rabczuk, "An adaptive singular ES-FEM for mechanics problems with singular field of arbitrary order," Computer Methods in Applied Mechanics and Engineering, vol. 253, pp. 252-273, 2013.

[79] N. Vu-Bac, H. Nguyen-Xuan, L. Chen et al., "A node-based smoothed extended finite element method (NS-XFEM) for fracture analysis," Computer Modeling in Engineering and Sciences, vol. 73, no. 4, pp. 331-355, 2011.

[80] N. Vu-Bac, H. Nguyen-Xuan, L. Chen et al., "A phantom-node method with edge-based strain smoothing for linear elastic fracture mechanics," Journal of Applied Mathematics, vol. 2013, Article ID 978026, 12 pages, 2013.

[81] A. Pandolfi, P. Krysl, and M. Ortiz, "Finite element simulation of ring expansion and fragmentation: the capturing of length and time scales through cohesive models of fracture," International Journal of Fracture, vol. 95, no. 1-4, pp. 279-297, 1999.

[82] T. Rabczuk, G. Zi, A. Gerstenberger, and W. A. Wall, "A new crack tip element for the phantom-node method with arbitrary 
cohesive cracks," International Journal for Numerical Methods in Engineering, vol. 75, no. 5, pp. 577-599, 2008.

[83] X.-P. Xu and A. Needleman, "Numerical simulations of fast crack growth in brittle solids," Journal of the Mechanics and Physics of Solids, vol. 42, no. 9, pp. 1397-1434, 1994.

[84] J. Fineberg, E. Sharon, and G. Cohen, "Crack front waves in dynamic fracture," International Journal of Fracture, vol. 121, no. 1-2, pp. 55-69, 2003.

[85] K. Ravi-Chandar, "Dynamic fracture of nominally brittle materials," International Journal of Fracture, vol. 90, no. 1-2, pp. 83102, 1998.

[86] E. Sharon, S. P. Gross, and J. Fineberg, "Local crack branching as a mechanism for instability in dynamic fracture," Physical Review Letters, vol. 74, no. 25, pp. 5096-5099, 1995.

[87] T. Rabczuk and G. Zi, "A meshfree method based on the local partition of unity for cohesive cracks," Computational Mechanics, vol. 39, no. 6, pp. 743-760, 2007.

[88] J. F. Kalthoff and S. Winkler, "Failure mode transition at high rates of shear loading," in Proceedings of the International Conference on Impact Loading and Dynamic Behavior of Materials, vol. 1, pp. 185-195, 1987.

[89] J. F. Kalthoff, "Modes of dynamic shear failure in solids," International Journal of Fracture, vol. 101, no. 1-2, pp. 1-31, 2000. 


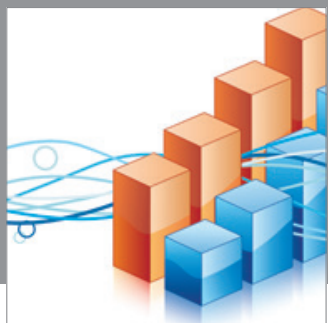

Advances in

Operations Research

mansans

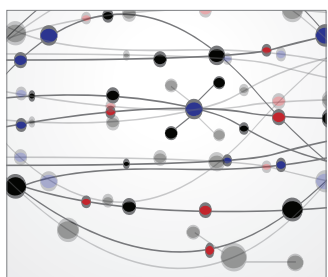

The Scientific World Journal
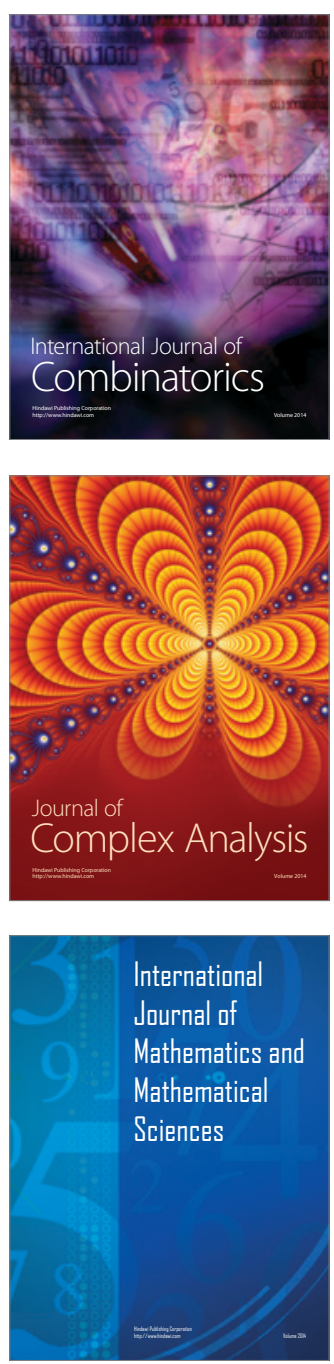
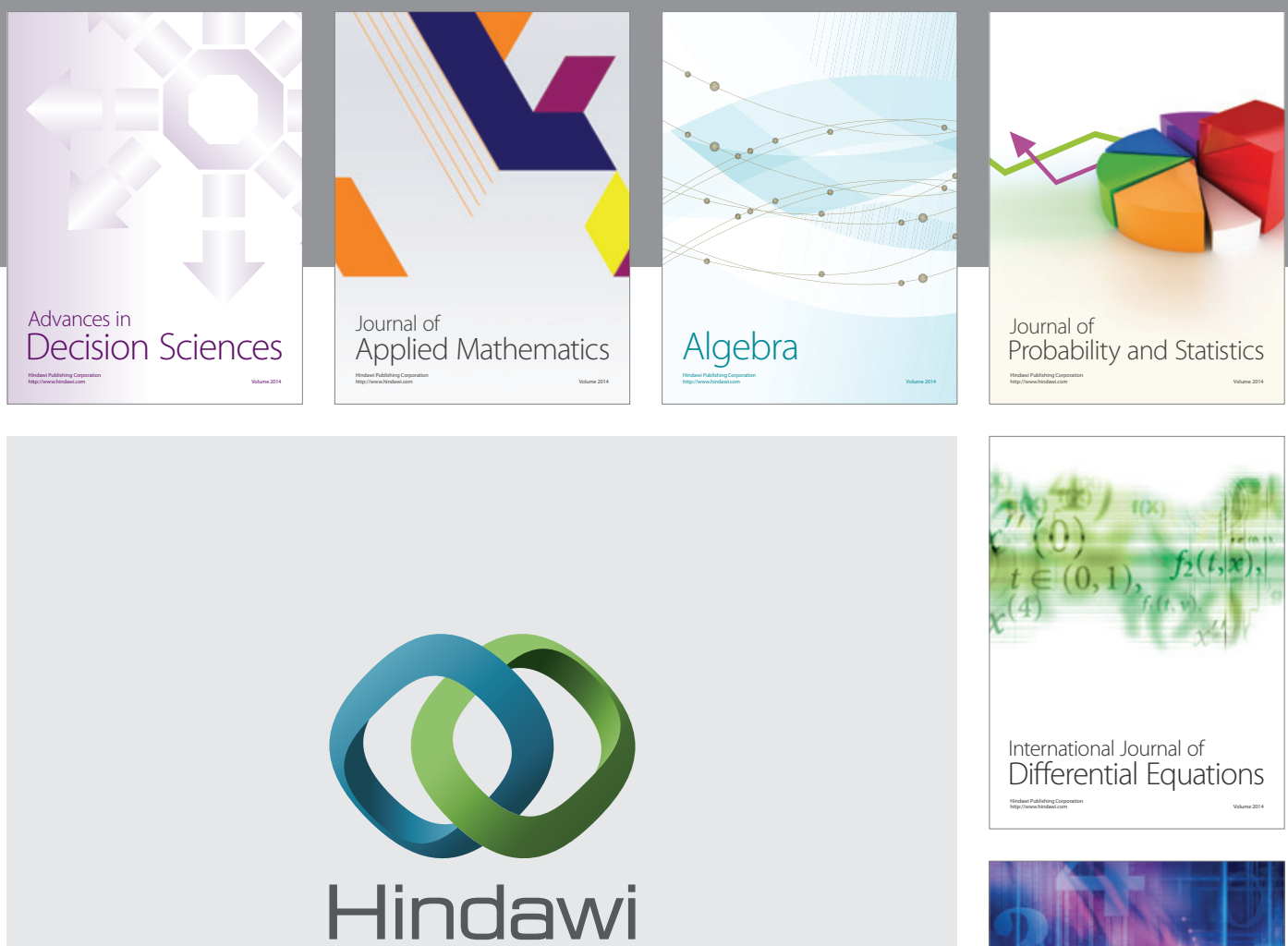

Submit your manuscripts at http://www.hindawi.com
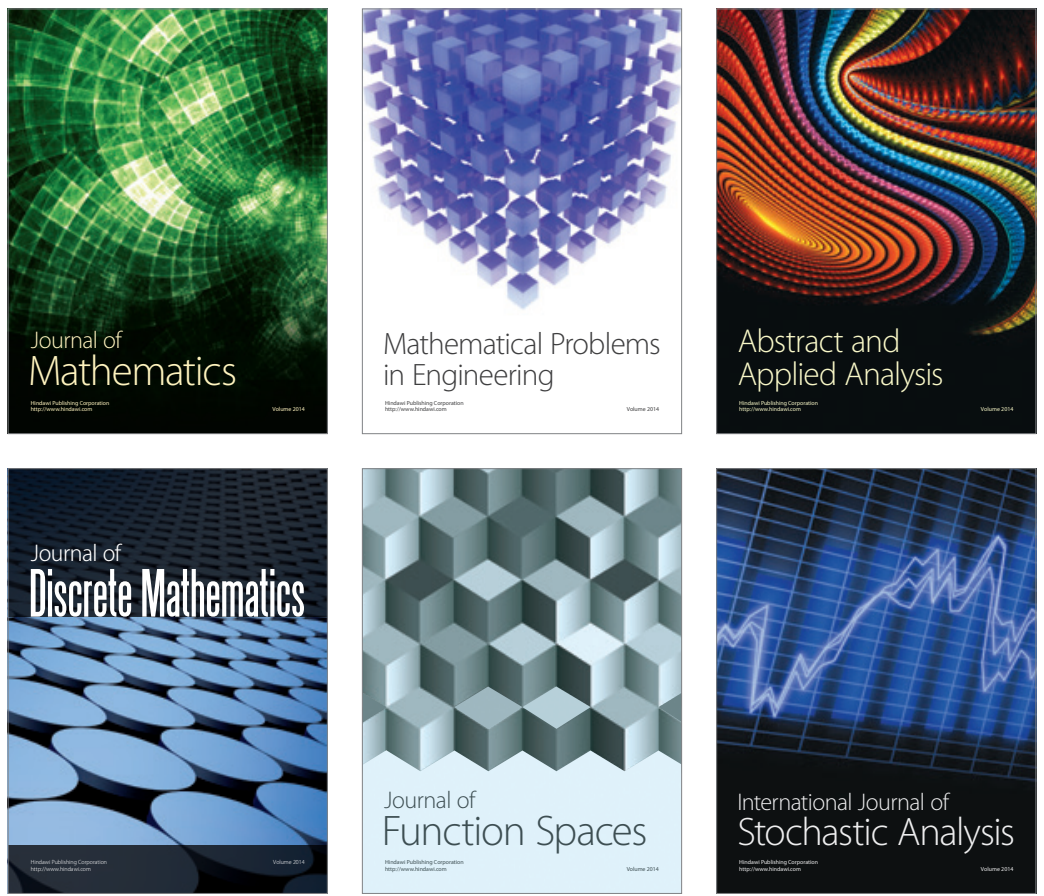

Journal of

Function Spaces

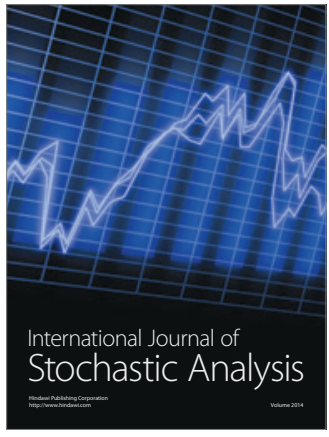

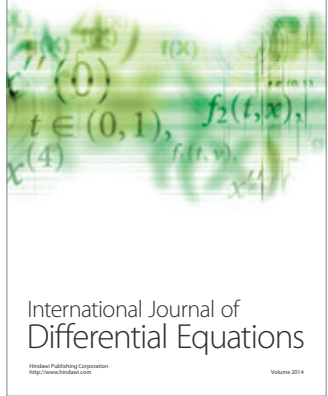
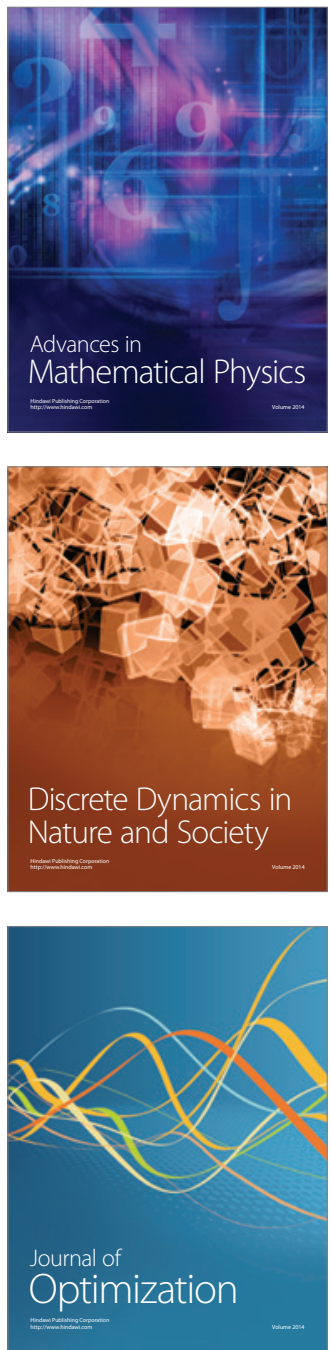